\title{
Vaginal Intraepithelial Neoplasia 3 in a Woman Without Simultaneous Cervical Intraepithelial Neoplasm
}

\author{
Eriko Hoshi-Sakamoto $^{\text {a, b }}$, Hisami Kiseki ${ }^{\text {a }}$, Shun-ichi Ikeda ${ }^{\text {a, b, c }}$
}

\begin{abstract}
In the presence of the uterus, it is rare that vaginal intraepithelial neoplasia (VAIN) alone exists. We described our experience with a case in which VAIN3 was found in the vaginal fornix without lesions in the uterine cervix. She had cervical intraepithelial neoplasm (CIN) 1 several years ago. After that, it disappeared. However, high-grade squamous intraepithelial lesion persisted cytologically. After hysterectomy was performed, VAIN3 was found in the vaginal fornix. CIN was not found in the cervix. We are apt to suspect endocervical lesions when Papanicolau (Pap) smear shows abnormalities and colposcopy fails to identify columnar epithelium. However, we need to keep in mind that the vaginal fornix may have a lesion. It may be difficult to assess the vaginal fornix in young women because of a large cervix. The presence of VAIN should also be considered when cytological abnormalities persist on Pap smears.
\end{abstract}

Keywords: Vaginal intraepithelial neoplasia; Human papilloma virus; Regression

\section{Introduction}

Vaginal intraepithelial neoplasia (VAIN) as well as cervical intraepithelial neoplasm (CIN) are premalignant lesions associated with human papilloma virus (HPV) [1]. VAIN is observed either when vaginal lesions are present simultaneously with cervical dysplasia or when VAIN appears in the vagina during follow-up after total hysterectomy for CIN or cervical cancer $[2,3]$. We here report a case in which VAIN3 was found in the vaginal fornix without lesions in the uterine cervix.

Manuscript submitted June 15, 2020, accepted June 23, 2020

Published online July 21, 2020

aDepartment of Obstetrics and Gynecology, Kohseichuo General Hospital, Tokyo, Japan

${ }^{b}$ Both authors contributed equally to this manuscript.

${ }^{\mathrm{c} C}$ Corresponding Author: Shun-ichi Ikeda, Department of Obstetrics and Gynecology, Kohseichuo General Hospital, 1-11-7, Mita, Meguro-ku 153-8581, Tokyo, Japan. Email: shiked@kohseichuo.jp

doi: https://doi.org/10.14740/jmc3532

\section{Case Report}

A 39-year-old gravida 5 para 2 woman received a gynecologic check-up by her family physician several years ago. Papanicolau (Pap) smear confirmed high-grade cervical intraepithelial neoplasm (HSIL). Although biopsy was performed using colposcopy, the pathological result was CIN1. Afterwards, she subsequently received regular Pap smears. HSIL persisted as the cytological result, but colposcopy showed no abnormal findings in the portio vaginalis. HPV type 16 was positive.

Her family physician considered that the lesion was in the cervical canal because no lesion could be observed in the portio vaginalis on colposcopy. He recommended that the patient should undergo conization. However, she firmly refused. She came to our hospital because she wanted a total hysterectomy. We also performed colposcopy. Squamous epithelium and a transformation zone were seen, but we did not detect columnar epithelium in the uterine cervix. We also proposed her to undergo cone biopsy. She strongly requested hysterectomy because HPV 16 was positive, HSIL was detected by cervical cytology, and she already had two children, so she had no desire for childbearing. We performed total abdominal hysterectomy (TAH) with bilateral salpingectomy (BSG). After surgery, the cervix was divided into 12 parts which were pathologically analyzed. Although no CIN was observed in the uterine cervix, dysplastic cells were found in five of 12 sections of the vaginal fornix. Three of five sections showed VAIN2, and VAIN3 was found in two sections (Fig. 1).

\section{Discussion}

We described our experience with a case of severe dysplasia in the vaginal fornix with no dysplasia in the cervix on hysterectomy. Two interesting points were found in this patient. One was mild dysplasia, which initially existed in the cervix. However, subsequent follow-up revealed that the lesion disappeared, and severe dysplasia was found in the vaginal fornix in the resected uterus. Second, it is often difficult to assess the vaginal fornix in young women because of a large portio vaginalis. This makes it difficult to detect VAIN.

In the female genitalia, squamous cell carcinoma and dysplasia usually occur in the uterine cervix. Reserve cells in the cervical transformation zone are critical for the pathogenesis of squamous cell carcinoma of the cervix and its precancerous lesions [4]. Although HPV is thought to be transmitted 


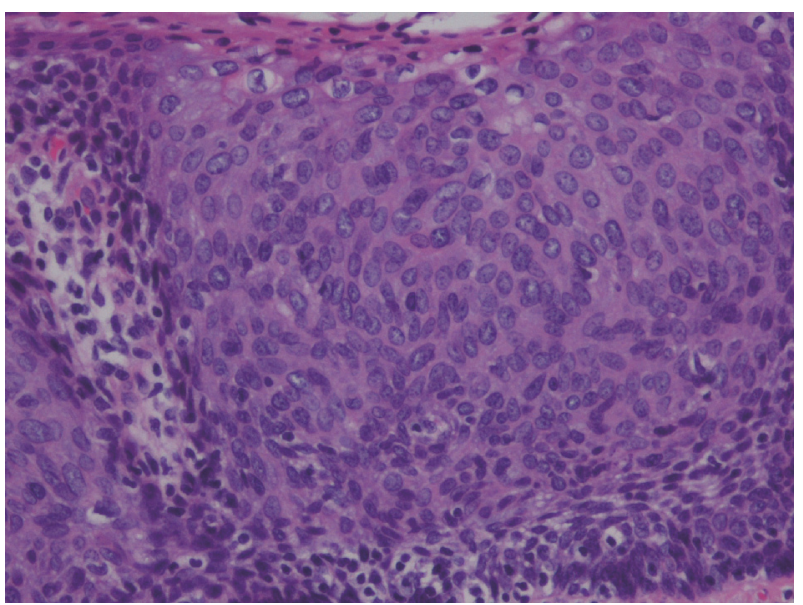

Figure 1. Severe atypia reaching the upper third of the squamous epithelium in the vaginal fornix (hematoxylin and eosin (H\&E) stain, $\times 20$ ).

from the epithelial damage site of basal cells or basal cell-like cells of immature squamous epithelium, the initial site of HPV infection is considered a transformation zone [5]. This is the reason why initial CIN rarely occurs outside the transformation zone.

The infected HPV genome becomes pathological CIN. The HPV genome, which exists as an episomal state in the basal cell nucleus, eventually causes spontaneous regression. After the integrated host cell acquires growth ability leading to clonal growth, spontaneous regression is difficult. It has been reported that the integration rate of HPV DNA into the host cell DNA is $27.8 \%$ for CIN1, 25-73.5\% for CIN2-3, and 81.6$81.8 \%$ for advanced dysplasia/intraepithelial carcinoma $[6,7]$. $\mathrm{CIN}$ is a pathological diagnosis. Therefore, it is not synonymous with the degree of CIN and genomic integration. CIN3 also has natural healing because it is related to the integration rate of CIN3.

We found that moderate to severe dysplasia was present only in the vaginal fornix after the uterus was removed.

Nearly all cases of VAIN are observed either when vaginal lesions are present with cervical dysplasia simultaneously or when VAIN appears in the vagina during follow-up after total hysterectomy for CIN or cervical cancer because latent HPV persistently infects the vagina and then causes VAIN. HPV infection takes the form of episomal to integrated, but it is eventually removed from human cells in the episomal form.

In our patient, dysplasia was found in the cervix at the first visit to another hospital. HPV infection thus existed not only in the vaginal fornix but also in the uterine cervix in our patient. This suggests that the HPV infection spread to the vaginal fornix after infection, and HPV in the cervix regressed in the episomal state. We speculated that the residual HPV of the vaginal fornix subsequently progressed to an integrated state and then progressed to severe dysplasia. Since HPV is known to infect reserve cells existing on the squamocolumnar (SC) junction, it is unlikely that it infects only the vaginal epithelium without infecting reserve cells in the SC junction.

We often experience difficulty in observing the vaginal fornix owing to the large cervix in young women. Harvey per- formed cervical measurements in 145 patients who underwent total hysterectomy [8]. Cervical diameter was shown to be associated with age, parity, and hormone status. The average diameter of the cervix was $3.5 \mathrm{~cm}$ in women younger than 55 years, but was $2.8 \mathrm{~cm}$ in women older than 55 years. Uterine cervical diameter was significantly larger in women with a history of birth or in menses than in women without these factors. A large cervix makes it difficult to observe the vaginal fornix. As a postpartum woman's vagina relaxes and protrudes into the speculum, observation of the vaginal fornix becomes more difficult. Our patient also had menstruation, a large cervix, and a history of childbirth. Therefore, observation of the vaginal fornix was difficult.

In conclusion, we experienced a case in which there was no lesion in the cervix and VAIN3 was found in the vaginal fornix. We are apt to suspect endocervical lesions when Pap smear shows abnormalities and colposcopy fails to identify columnar epithelium. However, we need to keep in mind that the vaginal fornix may have a lesion. If Pap smear shows abnormalities, but colposcopy shows no abnormalities in the cervix, it is necessary to check for vaginal fornix lesions. We consider that it is necessary to carefully observe the vagina including the vaginal fornix by colposcopy with the patient under anesthesia, depending on the situation.

\section{Acknowledgments}

None to declare.

\section{Financial Disclosure}

None to declare.

\section{Conflict of Interest}

None to declare.

\section{Informed Consent}

Informed consent was obtained from the patient prior to completing the case report.

\section{Author Contributions}

EHS and SiI contributed to the writing of the manuscript. EHS, $\mathrm{HK}$ and $\mathrm{SiI}$ reviewed and adjusted the manuscript prior to submission.

\section{Data Availability}

The data supporting the findings of this study are available from the corresponding author upon reasonable request. 


\section{References}

1. Frega A, Sopracordevole F, Assorgi C, Lombardi D, V DES, Catalano A, Matteucci E, et al. Vaginal intraepithelial neoplasia: a therapeutical dilemma. Anticancer Res. 2013;33(1):29-38.

2. Schockaert S, Poppe W, Arbyn M, Verguts T, Verguts J. Incidence of vaginal intraepithelial neoplasia after hysterectomy for cervical intraepithelial neoplasia: a retrospective study. Am J Obstet Gynecol. 2008;199(2):113 e111115.

3. Lamos C, Mihaljevic C, Aulmann S, Bruckner T, Domschke C, Wallwiener M, Paringer C, et al. Detection of human papillomavirus infection in patients with vaginal intraepithelial neoplasia. PLoS One. 2016;11(12):e0167386.

4. Doorbar J, Griffin H. Refining our understanding of cervical neoplasia and its cellular origins. Papillomavirus Res.
2019;7:176-179.

5. Lopez J, Ruiz G, Organista-Nava J, Gariglio P, GarciaCarranca A. Human papillomavirus infections and cancer stem cells of tumors from the uterine cervix. Open Virol J. 2012;6:232-240.

6. Cricca M, Morselli-Labate AM, Venturoli S, Ambretti S, Gentilomi GA, Gallinella G, Costa S, et al. Viral DNA load, physical status and E2/E6 ratio as markers to grade HPV16 positive women for high-grade cervical lesions. Gynecol Oncol. 2007;106(3):549-557.

7. Pirami L, Giache V, Becciolini A. Analysis of HPV16, 18,31 , and 35 DNA in pre-invasive and invasive lesions of the uterine cervix. J Clin Pathol. 1997;50(7):600604.

8. Harvey MA. The size of the cervix and its relationship with age and parity. Urogynaecologia International J. 2016;29:171. 